\title{
Caracterização morfológica de acessos de curauá do banco ativo de germoplasma da Embrapa Amazônia Oriental
}

\author{
Carolina Mariane Moreira ${ }^{1}$ \\ Suzan Kelly Vilela Bertolucci \\ Osmar Alves Lameira ${ }^{3}$ \\ Tainá Teixeira Rocha4 \\ Helena Botelho Andrade ${ }^{5}$ \\ Manuel Losada Gavilanes ${ }^{6}$ \\ José Eduardo Brasil Pereira Pinto7
}

\section{Resumo}

Neste trabalho, procurou-se avaliar a caracterização morfológica e agronômica de dois grupos de acessos de curauá (Ananas comosus var. erectifolius) do Banco Ativo de Germoplasma da Embrapa Amazônia Oriental. Os acessos foram avaliados de acordo com a idade das plantas, com 6 meses (15 acessos) e 12 meses (26 acessos). Os descritores utilizados foram: comprimento, largura, relação comprimento/largura, número e cor, das folhas. Os resultados foram submetidos à análise de variância e ao emprego do teste de Scott-Knott à análise de agrupamento e de componentes principais. A análise de variância resultou em diferenças significativas em ambos os grupos. Comparando, simultaneamente, as médias das variáveis respostas comprimento e relação comprimento/largura, observou-se que, para as plantas do grupo 1 , as maiores médias foram alcançadas nos acessos FMR 2600 e SRR 2500. Já para o grupo 2, incluem os acessos PEMATEC R, PAB 900, EMBRAPA R, PPR 400 e ANR 1200. No dendrograma de similaridade entre os 15 acessos de curauá, observou-se que há um grupo maior composto por 13 acessos constituído por 3 subgrupos e um grupo menor. No grupo 2, observou-se a formação de dois grandes grupos e vários subgrupos. A análise de componentes principais mostrou grupos semelhantes aos observados no dendrograma.

Palavras-chave: Análise de agrupamento. Descritores. Similaridade genética. Ananas comosus var. erectifolius.

\section{Introdução}

O curauá [Ananas comosus (L.) Merr. var. erectifolius (L. B. Smith) Coppens \& F. Leal] é uma espécie vegetal muito comum na Amazônia, pertencente à família Bromeliaceae, de porte herbáceo e,

\footnotetext{
1 Universidade Federal de Lavras, Campus Universitário, Caixa Postal 3037, CEP 37.200-000, Lavras-MG. E-mail: carolinammoreira@hotmail.com 2 Universidade Federal de Lavras, Campus Universitário, Caixa Postal 3037, CEP 37.200-000, Lavras-MG. E-mail: suzan@dag.ufla.br 3 Embrapa Amazônia Oriental, Travessa Dr. Enéas Pinheiro s/n, Caixa postal, 48 Belém, PA - Brasil, CEP 66.095-100. E-mail: osmar@cpatu.embrapa.br 4 Embrapa Amazônia Oriental, Travessa Dr. Enéas Pinheiros/n, Caixa postal, 48 Belém, PA-Brasil, CEP66.095-100. E-mail: tainarocha@yahoo.com.br 5 Universidade Federal de Lavras, Campus Universitário, Caixa Postal3037,CEP37.200-000, Lavras-MG. E-mail: heleninhaba@yahoo.com.br $6 \quad$ Universidade Federal de Lavras, Campus Universitário, Caixa Postal 3037, CEP 37.200-000, Lavras-MG. E-mail: gavilane@dbi.ufla.br 7 Universidade Federal de Lavras, Campus Universitário, Caixa Postal 3037, CEP 37.200-000, Lavras-MG. E-mail: jeduardo@dag.ufla.br - Autor para correspondência
} 
que apresenta duas variedades: a roxa e a branca (COPPENS; LEAL, 2003). A espécie tem como principal utilização no agronegócio a produção de fibras naturais. As fibras são empregadas em diversos setores como manufaturados, na produção de cordas, sacos e capacetes, papelaria, indústria têxtil, como sucedânea do linho, construção civil, itens automobilísticos e usos agrocomerciais (ARAÚJO, 2003).

Recentemente, na indústria automotiva, parte do revestimento interno de automóveis, freios, bancos, painéis, pára-choques e outros substitutos da fibra de vidro estão sendo confeccionados por materiais de origem natural, sendo que a fibra do curauá é uma das matérias primas empregadas. As principais características da fibra de curauá que possibilitam esses usos múltiplos são a resistência à tensão, peso reduzido e maciez (RAMALHO, 2005).

Estudo feito por Fujihashi e Barbosa (2002) demonstrou que o curauá também é considerado uma espécie medicinal por possuir propriedade antimicrobiana, o que lhe confere maior valor agregado na indústria têxtil.

A crescente demanda na utilização de fibras naturais deve-se, principalmente, ao aumento da preocupação mundial com a preservação do meio ambiente e a utilização de matéria prima renovável. Matérias-primas de origens renováveis, recicláveis e biodegradáveis são uma das alternativas para a produção de manufaturados ecologicamente corretos, em consequência do acúmulo nos descartes de materiais não biodegradáveis, os quais tendem a aumentar com o crescimento populacional nos centros urbanos. A substituição de materiais derivados do petróleo, na produção de compostos elastoméricos, por matéria-prima renovável vai ao encontro desses ideais. A fibra dessa planta tem alta resistência, maciez e peso reduzido. Há um grande interesse na busca por fibras naturais que possam substituir adequadamente as fibras sintéticas como, por exemplo, a fibra de vidro (SILVA et al., 2008).

Informações a respeito da diversidade genética de uma coleção de germoplasma servem para aumentar a eficiência dos trabalhos de melhoramento das espécies cultivadas. 0 estudo de divergência permite conhecer o grau da variabilidade genética das populações vegetais e subsidia a seleção de genitores geneticamente mais divergentes, que poderão ser utilizados em intercruzamentos com possibilidade de aumentar a probabilidade de recuperação de segregantes superiores em gerações avançadas (CRUZ; CARNEIRO, 2003).

Apesar da importância de se estudar a diversidade genética, não há relatos publicados de tais estudos em curauá para orientar a conservação e uso do germoplasma.

As técnicas de análises multivariadas têm sido empregadas para a quantificação da divergência genotípica e fenotípica em várias espécies (VIEIRA et al., 2010; NEIVA et al., 2011). Os métodos preditivos de diversidade genética têm sido bastante utilizados, sobretudo pelo fato de que, ao se basearem em diferenças morfológicas e fisiológicas dos genitores, dispensam a obtenção das combinações híbridas entre eles, o que é vantajoso, especialmente quando o número de genitores, cujas diversidades se deseja conhecer, é elevado.

Diante do grande interesse comercial que envolve a cultura do curauá e da falta de informações a respeito dessa espécie, a caracterização morfológica e agronômica dos acessos de um Banco de Germoplasma possibilita a diferenciação fenotípica entre eles, servindo como importante instrumento para a eliminação de duplicidades de acessos. Assim, trabalhos de caracterização e avaliação do germoplasma de curauá são fundamentais para a sua utilização mais eficiente nos trabalhos de meIhoramento, possibilitando a identificação de cultivares com características superiores e herdáveis.

Dessa forma, objetivou-se com este trabalho realizar a caracterização morfológica e agronômica de dois grupos com diferentes idades (6 e 12 meses) de acessos de curauá do Banco Ativo de Germoplasma da Embrapa Amazônia Oriental, Belém, PA. 


\section{Material e Métodos}

Neste estudo, foram utilizados acessos de curauá pertencentes à coleção de germoplasma da Embrapa Amazônia Oriental, localizado no município de Belém, PA, a $1^{\circ} 27^{\prime} 21^{\prime \prime}$ 'S de latitude e $48^{\circ} 30^{\prime} 14^{\prime \prime}$ 'W de longitude, com altitude de $10 \mathrm{~m}$ e temperatura média anual de $30^{\circ} \mathrm{C}$. A coleta dos dados foi realizada em julho de 2010, em acessos com 6 e 12 meses de idade, os quais foram divididos em dois grupos, de acordo com a coloração das folhas (branca e roxa). Os descritores avaliados foram: comprimento da maior folha $(\mathrm{cm})$, largura da maior folha $(\mathrm{cm})$, relação comprimento/largura e número de folhas por planta. Foram utilizadas em média cinco plantas por acesso para a coleta dos dados. Os acessos foram avaliados separadamente, de acordo com a idade das plantas: para 6 meses (grupo 1) e 12 meses (grupo 2), foram coletados dados de 15 e 26 acessos, respectivamente. O caráter cor da folha, por ser qualitativo, não entrou na análise estatística.

Exsicatas dos materiais trabalhados e coletados no Horto estão depositadas no Herbário IAN (Embrapa Amazônia Oriental), com os seguintes números: IAN 185100 - Ananas comosus var. erectifolius, Bromeliaceae (cuaruá-roxo) e IAN 185101 Ananas comosus var. erectifolius, Bromeliaceae (curauá-branco).

A fim de verificar a existência de variabilidade genética entre os acessos, os dados quantitativos foram submetidos à análise de variância e as médias comparadas pelo teste de Scott-Knott, ao nível de 5\% de probabilidade, utilizando o software Sisvar ${ }^{\circledR}$, versão 5.3 (FERREIRA, 2011).

Para realizar uma análise exploratória dos dados, foi feita uma Análise de Componente Principal (PCA-Principal Component Analysis). A PCA foi aplicada aos valores médios das variáveis de respostas de cada tratamento, sendo realizada uma PCA para cada grupo de acessos. Os dados foram pré-processados, empregando-se auto-escalonamento, e os resultados estão demonstrados pelos gráficos dos escores e dos pesos, com duas componentes principais (PC) explicadas pelas suas variâncias. Os cálculos foram feitos no software "MATLAB ${ }^{\circledR 7.5 " . ~}$

Na técnica de agrupamento, foi utilizada a distância Euclidiana como medida de dissimilaridade e, na delimitação dos grupos, foi utilizado o método hierárquico das médias das distâncias (UPGMA - Unweighted Pair Group Method with Arithmetic Mean).

\section{Resultados e Discussão}

A caracterização por meio de descritores morfológicos de germoplasma é requisito importante para analisar e quantificar a variabilidade genética de germoplasma. Vários trabalhos vêm sendo estudados com o objetivo de identificação de genótipos superiores ou seleções que atendam aos interesses dos produtores (SANTOS et al., 2012; MARTINS et al., 2012, RÊGO et al., 2011; SILVA et al., 2006; BLANK et al., 2004; MARTINELLO et al., 2002; DAROS et al., 2002; VEIGA et al., 2001)

Diferenças significativas $(p<0,05)$ foram observadas entre os acessos para todos os caracteres avaliados, o que indica variabilidade genética observada nas populações de Ananas comosus var. erectifolius, bem como seu potencial para uso em programas de melhoramento, visando à ampliação da base genética (Tabelas 1 e 2).

Por ter um grande potencial para produção de fibras naturais, folhas grandes e em grande quantidade são características de grande importância para os produtores. De modo geral, os acessos apresentaram características desejáveis para as variáveis que afetam a produção de fibras em curauá, pois em ambos os grupos observaram-se elevados valores de comprimento foliar (88,4 cm e 122,6 cm, respectivamente, para os grupos 1 e 2) e número de folhas por planta $(26,0$ e 28,4). 
Para o caráter cor da folha, o material avaliado foi polimórfico, visto que, tanto para acessos com 6 meses de idade quanto para acessos com 12 meses houve predominância de plantas com coloração foliar branca, acessos 9 e 15, respectivamente.

Os acessos com 6 meses de idade com coloração branca apresentaram, em geral, valores superiores para os descritores foliares. Por outro lado, as maiores médias nos acessos com 12 meses de idade foram obtidas nos de coloração roxa. A cor da folha foi a característica que menos proporcionou variabilidade entre os acessos.

Comparando, simultaneamente, as médias das variáveis de respostas comprimento e relação comprimento/largura, observou-se que, para as plantas do grupo 1, as maiores médias foram dos acessos FMR2600 e SRR2500, ambos com médias idênticas para as duas variáveis $(88,4 \mathrm{~cm}$ e 23,8 , respectivamente). Já para o grupo 2 , incluem os acessos PEMATEC R $(122,6 \mathrm{~cm}$ e 25,6$)$, PAB900 (109,5cm e 24,9), EMBRAPA R (105,3cm e 26,3), PPR400 $(104,2 \mathrm{~cm} \mathrm{e} \mathrm{24,3)} \mathrm{e} \mathrm{ANR1200}$ $(107,7 \mathrm{~cm}$ e 26,3$)$.

Com relação ao número de folhas, os acessos apresentaram valores médios de 19,6 e 18,9, respectivamente, para os grupos 1 e 2. Os acessos AMR2300 (24,5), FNR2100 (26,0), JPB1401 $(22,8)$, NLR2200 $(21,1)$ e SA1701 $(21,2)$ foram os que apresentaram as maiores médias para o grupo 1 . Para o grupo 2, os maiores valores médios observados foram para os acessos PEMATEC R (22,0), EMBRAPA R $(26,9)$, PPR400 $(28,4)$, TR300 (23,6), PEMATEC B $(20,4)$, ANR1200 $(26,7)$, AB101 $(22,6)$, MFR800 (22,3), BFR600 $(21,0)$ e ANB $1201(21,2)$. Resultados distintos dos deste estudo foram obtidos por Ramalho (2005), em estudos com essa espécie, o qual obteve entre 12 e 15 folhas, das quais foram extraídos cerca de $2 \mathrm{Kg}$ de fibras.

Tabela 1 Valores dos descritores foliares que caracterizam os acessos de curauá com idade de 6 meses (grupo 1) do Banco Ativo de Germoplasma da Embrapa Amazônia Oriental

\begin{tabular}{|c|c|c|c|c|c|c|}
\hline $\begin{array}{l}\text { Número do } \\
\text { acesso }\end{array}$ & Acesso & $\begin{array}{c}\text { Comprimento } \\
\text { (cm) }\end{array}$ & Largura (cm) & $\begin{array}{l}\text { Comp./ } \\
\text { largura }\end{array}$ & Número & Cor \\
\hline 15 & FMR2600 & $88,4 a^{*}$ & $3,7 b$ & $23,8 \mathrm{a}$ & $16,3 \mathrm{~b}$ & $B$ \\
\hline 14 & SRR2500 & $88,4 \mathrm{a}$ & $3,7 \mathrm{~b}$ & 23,8 a & $16,3 \mathrm{~b}$ & $B$ \\
\hline 11 & AMR2300 & $74,7 \mathrm{~b}$ & $4,5 \mathrm{a}$ & 16,6 b & $24,5 \mathrm{a}$ & $B$ \\
\hline 12 & FNR2100 & $74,5 \mathrm{~b}$ & 5,2 a & 14,4 b & $26,0 \mathrm{a}$ & $\mathrm{R}$ \\
\hline 13 & JPB1401 & $71,7 \mathrm{~b}$ & $4,8 \mathrm{a}$ & $14,9 \mathrm{~b}$ & $22,8 \mathrm{a}$ & $B$ \\
\hline 9 & NLR2200 & $67,0 \mathrm{~b}$ & $4,8 a$ & $13,95 b$ & $21,1 \mathrm{a}$ & $B$ \\
\hline 10 & SA1701 & $64,0 \mathrm{~b}$ & $4,7 \mathrm{a}$ & 13,6 b & $21,2 \mathrm{a}$ & $B$ \\
\hline 7 & NFB1901 & $62,2 \mathrm{~b}$ & $5,0 a$ & $12,5 \mathrm{~b}$ & $16,2 \mathrm{~b}$ & $B$ \\
\hline 8 & LAB1801 & $61,5 \mathrm{~b}$ & $4,5 a$ & 13,7 b & $20,5 \mathrm{a}$ & $\mathrm{R}$ \\
\hline 5 & EF1501 & $60,2 \mathrm{~b}$ & $4,2 b$ & 14,4 b & $18,8 \mathrm{~b}$ & $B$ \\
\hline 4 & HBR2400 & $56,3 \mathrm{~b}$ & $4,0 \mathrm{~b}$ & $14,0 \mathrm{~b}$ & $16,0 \mathrm{~b}$ & $\mathrm{R}$ \\
\hline 3 & NMB1301 & $56,1 \mathrm{~b}$ & $4,6 \mathrm{~b}$ & $12,2 \mathrm{~b}$ & $22,6 \mathrm{a}$ & $\mathrm{R}$ \\
\hline 6 & HBB2401 & $55,0 \mathrm{~b}$ & $4,7 \mathrm{a}$ & 11,7 b & $20,3 \mathrm{a}$ & $B$ \\
\hline 1 & JR1601 & $47,2 \mathrm{c}$ & $4,4 \mathrm{~b}$ & $10,8 \mathrm{~b}$ & $14,6 \mathrm{~b}$ & $\mathrm{R}$ \\
\hline \multirow[t]{3}{*}{2} & ASB201 & $42,7 \mathrm{c}$ & $4,3 \mathrm{~b}$ & $9,9 \mathrm{~b}$ & $17,0 \mathrm{~b}$ & $\mathrm{R}$ \\
\hline & Média geral & 66,2 & 4,5 & 14,7 & 19,6 & \\
\hline & CV (\%) & 17 & 12,5 & 17,9 & 23,3 & \\
\hline
\end{tabular}

*Médias seguidas de letras iguais, nas colunas, não diferem entre si pelo teste de Scott-Knott $(p \leq 0,05)$. B - folha branca/ R - folha roxa.

Fonte: Elaboração dos autores 
Tabela 2 Valores dos descritores foliares que caracterizam os acessos de curauá com idade de 12 meses (grupo 2) do Banco Ativo de Germoplasma da Embrapa Amazônia Oriental

\begin{tabular}{|c|c|c|c|c|c|c|}
\hline $\begin{array}{c}\text { Número do } \\
\text { acesso }\end{array}$ & Acesso & $\begin{array}{c}\text { Comprimento } \\
(\mathrm{cm})\end{array}$ & Largura $(\mathrm{cm})$ & $\begin{array}{l}\text { Comp./ } \\
\text { largura }\end{array}$ & Número & Cor \\
\hline 1 & PEMATEC R & $122,6 a^{*}$ & $4,7 a$ & $25,6 a$ & $22,0 \mathrm{a}$ & $\mathrm{R}$ \\
\hline 2 & PAB900 & 109,5 a & $4,4 \mathrm{a}$ & 24,9 a & $14,0 \mathrm{~b}$ & $\mathrm{R}$ \\
\hline 3 & DR500 & $106,7 \mathrm{a}$ & $5,0 \mathrm{a}$ & $21,3 \mathrm{~b}$ & $24,4 \mathrm{a}$ & $\mathrm{R}$ \\
\hline 4 & EMBRAPA R & 105,3 a & 4,0 a & 26,3 a & 26,9 a & $\mathrm{R}$ \\
\hline 5 & PPR400 & $104,2 \mathrm{a}$ & 4,3 a & $24,3 \mathrm{a}$ & 28,4 a & $\mathrm{R}$ \\
\hline 6 & TR300 & $101,4 \mathrm{a}$ & 4,6 a & $22,0 \mathrm{~b}$ & 23,6 a & $\mathrm{R}$ \\
\hline 7 & PEMATEC B & $93,8 \mathrm{~b}$ & $3,8 \mathrm{~b}$ & 24,7 a & 20,4 a & B \\
\hline 8 & ANR1200 & $107,7 \mathrm{a}$ & 4,1 a & $26,3 a$ & 26,7 a & $\mathrm{R}$ \\
\hline 9 & AB101 & $85,2 \mathrm{~b}$ & $3,6 \mathrm{~b}$ & $23,8 \mathrm{a}$ & 22,6 a & B \\
\hline 10 & NVR1000 & $93,0 \mathrm{~b}$ & $3,6 \mathrm{~b}$ & $25,8 \mathrm{a}$ & 17,7 b & $\mathrm{R}$ \\
\hline 11 & RSB1101 & $72,8 \mathrm{c}$ & $4,0 \mathrm{a}$ & $18,5 \mathrm{~b}$ & 16,7 b & $B$ \\
\hline 12 & MFR800 & $86,0 \mathrm{~b}$ & $4,1 \mathrm{a}$ & $21,0 \mathrm{~b}$ & $22,3 \mathrm{a}$ & $\mathrm{R}$ \\
\hline 13 & RSR1100 & $107,0 \mathrm{a}$ & $4,8 \mathrm{a}$ & $22,1 \mathrm{~b}$ & 14,8 b & $\mathrm{R}$ \\
\hline 14 & BFR600 & $74,7 \mathrm{c}$ & $3,9 a$ & $19,3 \mathrm{~b}$ & 21,0 a & $\mathrm{R}$ \\
\hline 15 & ANB1201 & $88,1 \mathrm{~b}$ & $3,4 \mathrm{~b}$ & $25,9 a$ & 21,2 a & B \\
\hline 16 & NVB1001 & $63,9 \mathrm{c}$ & $3,1 \mathrm{~b}$ & $20,7 \mathrm{~b}$ & $14,0 \mathrm{~b}$ & B \\
\hline 17 & PAB901 & $78,97 \mathrm{c}$ & $3,7 b$ & $21,4 b$ & $17,5 \mathrm{~b}$ & B \\
\hline 18 & EMBRAPA B & $78,7 \mathrm{c}$ & $3,6 b$ & $21,9 \mathrm{~b}$ & $15,0 \mathrm{~b}$ & B \\
\hline 19 & PPB401 & $68,8 \mathrm{c}$ & $3,5 b$ & $19,6 \mathrm{~b}$ & 18,8 b & B \\
\hline 20 & MFB801 & $62,7 \mathrm{c}$ & $3,2 \mathrm{~b}$ & $19,6 \mathrm{~b}$ & 18,3 b & B \\
\hline 21 & DB501 & $51,3 d$ & $3,0 \mathrm{~b}$ & $17,1 \mathrm{c}$ & $13,3 \mathrm{~b}$ & B \\
\hline 22 & MB002 & $67,2 \mathrm{c}$ & $3,2 b$ & $21,0 \mathrm{~b}$ & $18,0 \mathrm{~b}$ & B \\
\hline 23 & TB301 & $62,1 \mathrm{c}$ & $3,7 b$ & $16,8 \mathrm{c}$ & 16,2 b & B \\
\hline 24 & CB201 & $46,8 d$ & $3,0 \mathrm{~b}$ & $15,6 \mathrm{c}$ & 13,8 b & B \\
\hline 25 & BB701 & $43,0 \mathrm{~d}$ & $2,9 \mathrm{~b}$ & $14,8 \mathrm{c}$ & $9,2 \mathrm{~b}$ & B \\
\hline \multirow[t]{3}{*}{26} & BFB601 & $39 d$ & $2,2 \mathrm{~b}$ & $17,8 \mathrm{c}$ & $9,5 \mathrm{~b}$ & $\mathrm{~B}$ \\
\hline & Média geral & 80,8 & 3,7 & 20,7 & 18,9 & \\
\hline & CV (\%) & 25,6 & 17,6 & 17,1 & 28 & \\
\hline
\end{tabular}

*Médias seguidas de letras iguais, nas colunas, não diferem entre si pelo teste de Scott-Knott $(p \leq 0,05)$. Bfolha branca/ R- folha roxa.

Fonte: Elaboração dos autores.

Pela análise do dendrograma obtido com base na distância Euclidiana, pode-se observar pela distância média entre os genótipos que há um grupo maior composto por 13 acessos constituídos por 3 subgrupos e um grupo menor composto pelos acessos 1 e 2 (Figura 1). Martinell et al. (2002) também trabalharam com as médias dos dados morfológicos em três componentes principais. Observa-se pelo dendrograma que os acessos 1 e 15 foram os mais contrastantes e os acessos 1 e 2 os mais similares. Os resultados apresentados indicam que, num programa de melhoramento, os acessos dos 3 subgrupos ( 3 a 7; 8, 9, 10, 12 e 13; 11, 14 e 15) que tiveram as maiores similaridades, a fim de maximizar a heterose, os componentes dentro de cada grupo não deveriam ser cruzados entre si. 


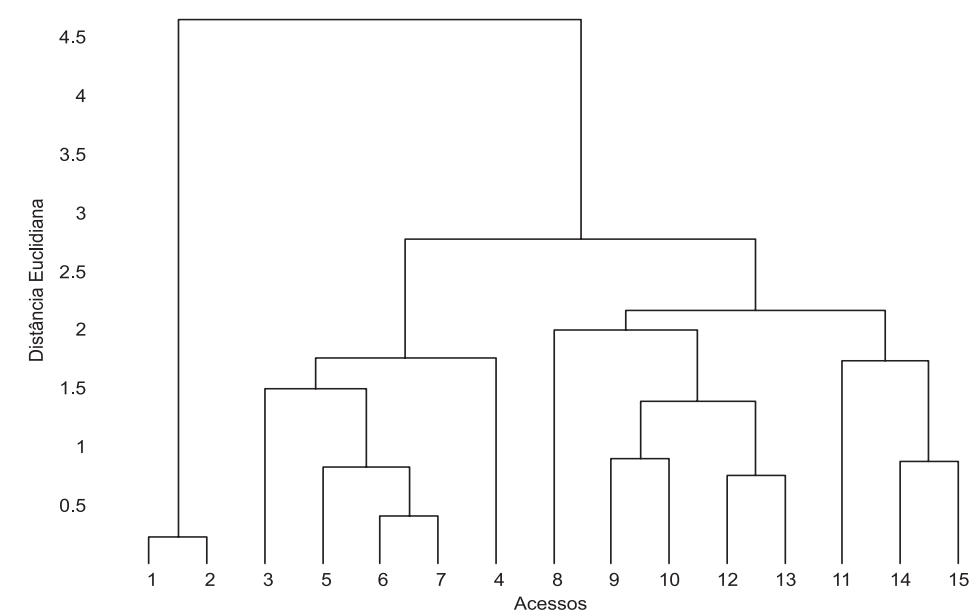

$\begin{array}{lccccc}1 & \text { JR1601 } & 6 & \text { HBB2401 } & 11 & \text { AMR2300 } \\ 2 & \text { ASB201 } & 7 & \text { NFB1901 } & 12 & \text { FNR2100 } \\ 3 & \text { NMB1301 } & 8 & \text { LAB1801 } & 13 & \text { JPB1401 } \\ 4 & \text { HBR2400 } & 9 & \text { NLR2200 } & 14 & \text { SRR2500 } \\ 5 & \text { EF1501 } & 10 & \text { SA1701 } & 15 & \text { FMR2600 }\end{array}$

Figura 1 Agrupamento dos acessos de curauá com idade de seis meses (grupo1) pelo método UPGMA, com base na diversidade genética expressa pela Distância Euclidiana média.

Fonte: Elaboração dos autores.

Pela análise de componentes principais utilizando quatro caracteres, a variância acumulada pelos dois primeiros componentes foi de $94,22 \%$ da variação total observada, visto que a primeira componente principal explica 59,62\% e a segunda, 34,60\% (Figura 2). Segundo Mardia, Kent e Bibby (1979), se os primeiros componentes acumularem uma porcentagem relativamente alta da variação total, em geral, acima de $80 \%$, eles explicam satisfatoriamente a variabilidade manifestada entre os indivíduos avaliados.
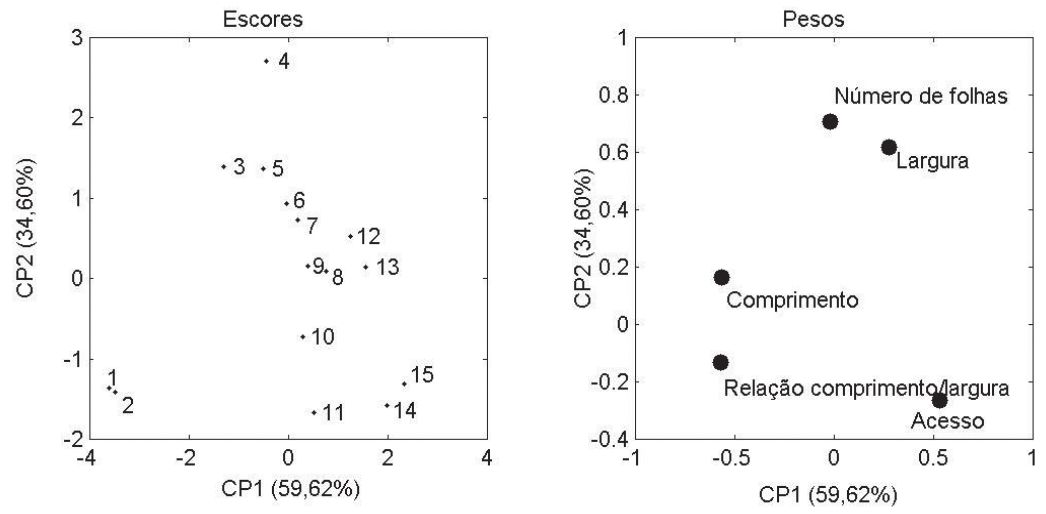

$\begin{array}{lccccc}1 & \text { JR1601 } & 6 & \text { HBB2401 } & 11 & \text { AMR2300 } \\ 2 & \text { ASB201 } & 7 & \text { NFB1901 } & 12 & \text { FNR2100 } \\ 3 & \text { NMB1301 } & 8 & \text { LAB1801 } & 13 & \text { JPB1401 } \\ 4 & \text { HBR2400 } & 9 & \text { NLR2200 } & 14 & \text { SRR2500 } \\ 5 & \text { EF1501 } & 10 & \text { SA1701 } & 15 & \text { FMR2600 }\end{array}$

Figura 2 Representação gráfica da análise de componentes principais de 15 acessos de curauá com idade de seis meses (grupo 1) a partir das quatro variáveis.

Fonte: Elaboração dos autores 
A análise de componentes principais mostrou grupos semelhantes aos observados no dendrograma da Figura 1. Observa-se no gráfico de escores (Figura 2) a separação marcante dos acessos 1 e 2, em relação aos demais. Também nota-se pelo gráfico de escores (Figura 2) que houve a formação de subgrupos característicos daqueles do dendrograma (Figura 1), como os que unem os acessos 3, 5, 6, 7 e 11, 14 e 15. Pela análise do gráfico de pesos sobre o de escores (Figura 2), observa-se que os caracteres que determinaram a separação desses dos últimos subgrupos foram o número e a largura das folhas.

Como observado na Tabela 1, houve diferença expressiva entre os acessos 14 e 15 e entre 1 e 2. Assim, comparando esse dado com a análise de componentes principais (Figura 2), o caractere de maior encargo foi dado pela relação comprimento/largura.

No dendrograma de similaridade (Figura 3), obtido pela distância Euclidiana, entre os 26 acessos de curauá (grupo 2) observou-se a formação de dois grandes grupos. Dentro deles, observa-se a formação de vários subgrupos, sendo um subgrupo formado por 14 acessos e o outro por 12. Observa-se pelo dendrograma que os acessos MFB 801 e RSR 1100 foram os mais contrastantes. Já os acessos MFB 801e MB 002, os mais próximos geneticamente.

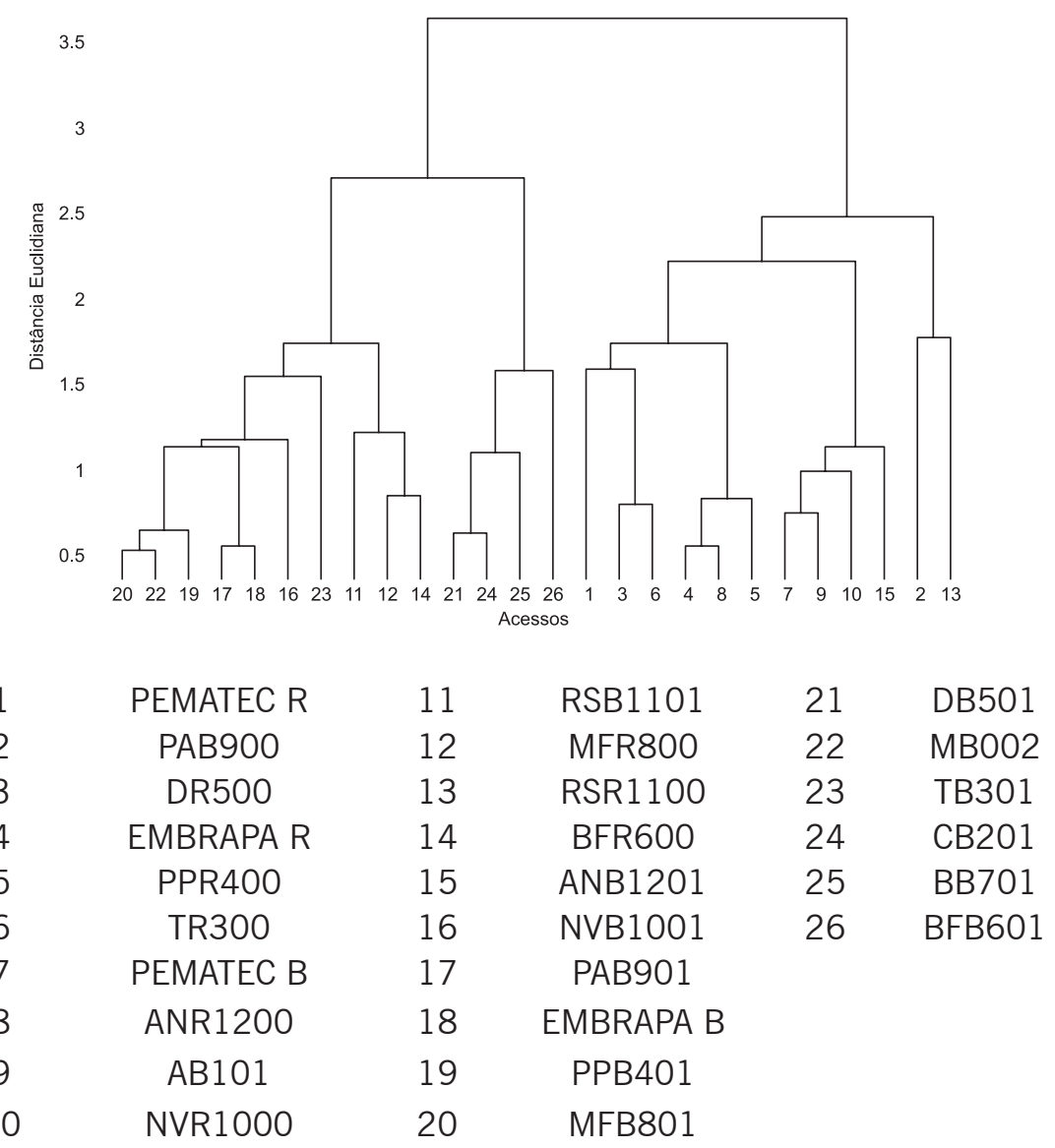

Figura 3 Agrupamento dos acessos de curauá com idade de doze meses pelo método UPGMA, com base na diversidade genética expressa pela distância euclidiana média.

Fonte: Elaboração dos autores. 
Foi obtida a representação gráfica das 4 variáveis e a distribuição dos 26 acessos sobre os dois primeiros componentes principais, e a variância acumulada pelos dois primeiros componentes foi de $90,10 \%$ da variação total observada, já que a primeira componente principal explica $80,11 \%$ e a segunda 9,99\% (Figura 4).
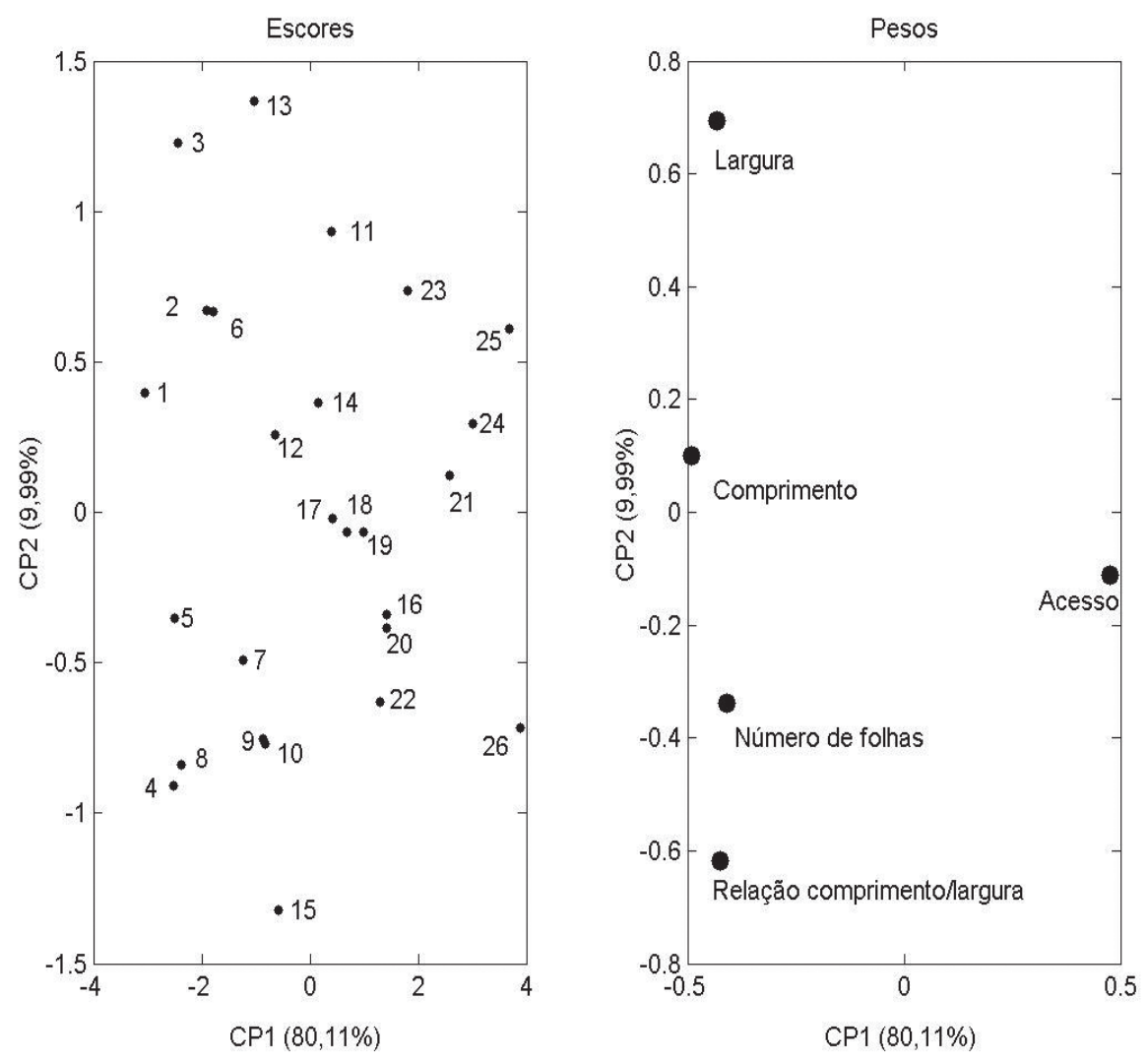

$\begin{array}{cccccc}1 & \text { PEMATEC R } & 11 & \text { RSB1101 } & 21 & \text { DB501 } \\ 2 & \text { PAB900 } & 12 & \text { MFR800 } & 22 & \text { MB002 } \\ 3 & \text { DR500 } & 13 & \text { RSR1100 } & 23 & \text { TB301 } \\ 4 & \text { EMBRAPA R } & 14 & \text { BFR600 } & 24 & \text { CB201 } \\ 5 & \text { PPR400 } & 15 & \text { ANB1201 } & 25 & \text { BB701 } \\ 6 & \text { TR300 } & 16 & \text { NVB1001 } & 26 & \text { BFB601 } \\ 7 & \text { PEMATEC B } & 17 & \text { PAB901 } & & \\ 8 & \text { ANR1200 } & 18 & \text { EMBRAPA B } & & \\ 9 & \text { AB101 } & 19 & \text { PPB401 } & & \\ 10 & \text { NVR1000 } & 20 & \text { MFB801 } & \end{array}$

Figura 4 Representação gráfica da análise de componentes principais de 26 acessos de curauá com idade de doze meses a partir das quatro variáveis.

Fonte: Elaboração dos autores.

Resultados semelhantes foram encontrados por Costa et al. (2002), em estudo com curauá. Utilizando marcadores RAPD, os autores observaram que os acessos PAB900 e RSR1100 também apresentaram alta distância genética em relação aos outros grupos de acessos. 
Os acessos MFB801 e MB002 estão próximos no gráfico de escores (Figura 4), assim como observado no dendrograma (Figura 3). 0 dendrograma e a análise de componentes principais também corroboram quanto à separação do acesso RSR1100.

A representação gráfica da análise de componentes principais dos 15 acessos com 6 meses de idade mostrou a distribuição dos acessos de forma mais semelhante à apresentada no agrupamento UPGMA do que a representação gráfica dos componentes principais dos 26 acessos de 12 meses de idade.

\section{Conclusões}

De modo geral, os resultados podem ser úteis na identificação de populações divergentes de curauá, a fim de direcionar os cruzamentos entre acessos classificados em grupos distintos e com genótipos favoráveis para produção de fibras.

Embora a caracterização morfo-agronômica seja vista como uma medida indireta da variabilidade total dos acessos, neste trabalho ela foi capaz de detectar acentuada diversidade entre todos os caracteres e entre todos os acessos. Entretanto, vale ressaltar a importância da avaliação do germoplasma do curauá para subsidiar programas de melhoramento.

\section{Agradecimentos}

Os autores agradecem à Fundação de Amparo à Pesquisa do Estado de Minas Gerais (FAPEMIG), Coordenação de Aperfeiçoamento de Pessoal de Nível Superior (CAPES), Conselho Nacional de Desenvolvimento Científico e Tecnológico (CNPq) pelo auxílio financeiro e concessão de bolsas de estudos e de produtividade.

\section{Morphologic characterization of curaua accesses from the active germplasm bank of the Eastern Amazon Embrapa}

\section{Abstract}

This work had the purpose to carry out the morphological and agronomical characterization of two curaua accesses groups from active germplasm bank of Eastern Amazon Embrapa. Accesses were evaluated according to the plants age for 6 months with 15 accesses and 12 months with 26 accesses. List of descriptors: leaf (length, width, length / width, number and color).The results were submitted to analyses of variance and Scott-Knott test $(p<0.05)$ and analyses of group and main components. The analyses of variance showed significant differences in both groups. Comparing, simultaneously, the average of variables responses length and length/width observed that plants from group 1 the higher means accesses FMR 2600 and SRR 2500. For the group 2 the accesses PEMATEC R, PAB 900, EMBRAPA R, PPR 400 and ANR 1200 were the highest. Dendrogram among the 15 curaua accesses could be observed that there is one larger group with 13 accesses with 3 subgroup and one smaller group. For group 2 it was observed the formation of two larger groups with many subgroups. The analyze of main components showed similar groups as showed in the dendrogram.

Keywords: Cluster analysis. Descriptors. Genetic similarity. Ananas comosus var. erectifolius 


\section{Referências}

ARAÚJO, C. R. Cinética de decomposição térmica de compósitos poliméricos com fibras de curauá. 2003. 220 p. Tese (Doutorado em Tecnologia de Processos Químicos e Bioquímicos) - Escola de Química, Universidade Federal do Rio de Janeiro, Rio de Janeiro, 2003.

BLANK, A. F.; FILHO, J. L. S. C.; NETO, A.L.S.; ALVES, P. B.; BLANK, M. F. A.; MANN, R. S.; MENDONÇA, M. C. Caracterização morfológica e agronômica de acessos de manjericão e alfavaca. Horticultura Brasileira, Brasília, v.22, p.113-116, 2004.

COPPENS, G.; LEAL, F. Morphology, anatomy and taxonomy. In: BARTHOLOMEW, D. P.; PAULL, R.E.; ROHRBACH,K.G. (Eds.). The pineapple: botany, production and uses. Wallingford: CABI, 2003. Disponível em: <http://home.tiscali.nl/leodg/ht/lists-species2000+html>. Acesso em: 01 jun. 2010.

COSTA, M. R.; LAMEIRA, O. A.; YOSBINO, V. C. Caracterização genética do curauá (Ananas erectifolius) através de marcadores RAPD. Biotecnologia Ciência \& Desenvolvimento, Brasília, v.5, p.28-30, 2002.

CRUZ, C. D.; CARNEIRO, P. C. S. Modelos biométricos aplicados ao melhoramento genético. Viçosa: Editora UFV, MG, 2003. v.2, 585 p.

DAROS, M.; JÚNIOR, A. T. A.; PEREIRA, T. N. S.; LEAL, N. R.; FREITRAS, S. P.; SEDIYMA, T. Caracterização morfológica de acessos de batata-doce. Horticultura Brasileira, Brasília, v.20, p.43-47, 2002.

FERREIRA, D. F. SISVAR 5.3: sistema de análise estatística. Lavras: UFLA/DEX, 2011. Software.

FUJIHASHI, G. A.; BARBOSA, W. L. R. Ananas erectifolius (curauá): padronização dos extratos, frações e do material vegetal. Revista Científica da UFPA, Belém, v.3, p. 1-6, 2002.

GALATE, R. S.; MOTA, M. G. C.; GAIA, J. M. D.; COSTA, M. S. S. Caracterização morfoagronômica de germoplasma de açaizeiro no nordeste paraense. Revista Brasileira de Fruticultura, v.34, p.540$545,2012$.

MARDIA, K. V.; KENT, J. T.; BIBBY, J. M. Multivariate analysis. London: Academic, 1979. 521 p.

MARTINELlO, G. E.; LEAL, N. R.; JÚNIOR, A. T. A.; PEREIRA, M. G.; DAHER, R. F. Divergência genética em acessos de quiabeiro com base em marcadores morfológicos, Horticultura Brasileira, Brasília, v.20, p.52-58, 2002.

MARTINS, C. P. S.; MELO, M. T. P.; HONÓRIO, I. C. G.; D’ÁVILA, V. A.; CARVALHO JÚNIOR, W. G. O. Caracterização morfológica e agronômica de acessos de jambu (Spilanthes oleracea L.) nas condições do Norte de Minas Gerais. Revista. Brasileira de Plantas medicinais, v.14, p.410-413, 2012.

NEIVA, I. P.; JÚNIOR, V. C. A.; VIANA, D. J.; FIGUEIREDO, J. A.; FILHO, C. V.; PARRELLA, R. A.; SANTOS, J. B. Caracterização morfológica de acessos de batata-doce do banco de germoplasma da UFVJM, Diamantina. Horticultura Brasileira, Brasília, v.29, p.537-541, 2011.

RAMALHO, E. A folha amazônica que virou arte. 2005. Disponível em: <http://www.rfi.fr/actubr/ articles/068/article_124.asp>. Acesso em: 15 out. 2010. 
RÊGO, E. R.; RÊGO, M. M.; MATOS, I. W. F.; BARBOSA, L. A. Morphological and chemical characterization of fruits of Capsicum spp. Accessions. Horticultura Brasileira, Brasília, v.29, p.364-371, 2011.

SILVA, R. V.; AQUINO, E. M. F.; RODRIGUES, L. P. S. ; BARROS, A. R. F. Desenvolvimento de um compósito laminado híbrido com fibras natural e sintética. Revista Matéria, v.13, p.154-161, 2008.

SILVA, M. L.; Queiróz, M. A.; FERREIRA, M. A. J. F.; BUSO, G. S. C. Caracterização morfológica e molecular de acessos de melancia. Horticultura Brasileira, Brasília, v.24, p.405-409, 2006.

VEIGA, R. F. A.; VOLTAN, R. B. Q.; VALLS, J. F. M.; FÁVERO, A. P.; BARBOSA, W.Caracterização morfológica de acessos de germoplasma de quatro espécies brasileiras de amendoim-silvetre. Bragantia, Campinas, v.60, p.167-176, 2001.

VIEIRA, L. D. J.; SANTOS, L. R.; CASTELEN, M. S.; JUNGHANS, D. T. Caracterização morfológica de acessos de abacaxizeiro. Revista Eletrônica da FAINOR, Vitória da Conquista, v.3, p.68-77, 2010. Disponível em: <http://srv02.fainor.com.br/revista/index.php/memorias/article/viewFile/74/69>. Acesso em: 15 dez. 2010.

\section{Histórico}

Submetido em: 19/12/2013

Aceito em: 21/07/2014 